\title{
Escuela y estratificación social en Chile: ¿cuál es el rol de la municipalización y la educación particular subvencionada en la reproducción de la desigualdad social?
}

\author{
School and social stratification in Chile: what is the role of "municipalización" \\ and the subsidized private education in the reproduction of social inequalities? \\ Escola e estratificação social no Chile: qual é o papel da municipalização e \\ do ensino privado subvencionado na reprodução da desigualdade social?
}

\section{Ismael Puga}

Humboldt Universität zu Berlin. Fono: +49-1-742774823.

E-mail: ismael.puga.rayo@cms.hu-berlin.de

\section{RESUMEN}

Se presenta un análisis empírico del impacto de la municipalización y de la diferenciación entre escuelas públicas y particular subvencionadas (PS) sobre el logro laboral futuro de los individuos, a partir de la información contenida en la encuesta EMSC 2001. Los resultados sugieren concordancia con estudios previos en que la educación PS no proporciona mejores resultados pedagógicos que su contraparte una vez controlado el origen social de los estudiantes, pero indican además que la segregación de éstos en diversas modalidades aporta a la reproducción de la desigualdad a través de diversos mecanismos indirectos. Estos mecanismos, se propone, pueden rastrearse hasta la segregación de redes y capital social, la distribución diferenciada de expectativas, y el establecimiento de credenciales simbólicas diferenciadas que son reconocidas como tales en el mercado laboral. Las escuelas PS, sin aportar sustantivamente al logro educativo, generan una mayor desigualdad de oportunidades escolares y laborales.

Palabras clave: municipalización, educación particular subvencionada, estratificación, desigualdad educacional.

\begin{abstract}
Using data from the survey EMSC 2001, the paper presents an empirical analysis of the impact of "municipalización" and the distinction between public and particular-subsidized (PS) schools into the future occupational achievement of individuals. The results seem concomitant with previous research which shows that PS schooling does not provide better pedagogic results than its counterpart once the students' social origins are controlled, and further provide evidence for the effect of segregating students into different modalities of schooling into greater reproduction of social inequalities trough other indirect mechanisms. These mechanisms, I propose, should be tracked back to the segregation of networks and social capital, the differentiation as well of expectatives, and the establishment of distinct symbolic credentials which are recognized as such in the labor market. The PS schools, without really contributing to educational outputs, do generate greater inequelities of educational and occupational opportunities.
\end{abstract}

Key words: "municipalización”, private-subsidized education, stratification, educational inequality.

\section{RESUMO}

Apresenta-se uma análise empírica do impacto da municipalização e da diferenciação entre escolas públicas e particulares subvencionadas (PS), a partir de dados da pesquisa EMSC 2001, referentes à busca de futuro emprego pelos sujeitos. Os resultados não diferem de anteriores, os quais revelaram que a educação PS não fornece resultados pedagógicos melhores do que a sua contraparte, constatada a origem social dos estudantes. No entanto, os resultados indicam que a segregação em variadas formas contribui para a reprodução da desigualdade social 
por meio de outros mecanismos indiretos. Neste artigo, postula-se que estes mecanismos podem ser atribuídos à segregação de redes e capital social, à distribuição diferencial das expectativas e ao estabelecimento de diferentes credenciais simbólicas que são reconhecidas como tal no mercado de trabalho. As escolas PS, além de não fornecerem importantes avanços educacionais, geram, por outro lado, grandes desigualdades de oportunidades educacionais e de emprego.

Palavras chave: municipalização, ensino privado subvencionado, estratificação, desigualdade educacional.

\section{INTRODUCCIÓN}

La desigualdad es uno de los tópicos característicos de las sociedades latinoamericanas. En particular, la sociedad chilena de las últimas décadas destaca por sus altísimos niveles de desigualdad en los ingresos en el contexto de un acelerado crecimiento económico. Esta desigualdad, que ha vuelto a incrementarse de acuerdo a la última encuesta CASEN 2009, debe comprenderse necesariamente en asociación al modelo de desarrollo económico impulsado a partir de la década de 1970, que impulsó una progresiva liberalización económica y la privatización de diversos servicios anteriormente en manos públicas.

Si existe consenso respecto de la desigualdad de resultados en Chile, el panorama en términos de estratificación y movilidad social es algo más complejo. El gran crecimiento de la escolaridad, particularmente a partir de la década de 1990, y en general la transformación productiva del país han dado como resultado mayores índices de movilidad social intergeneracional. Esta movilidad social de tipo estructural, sin embargo, no modifica necesariamente de modo lineal las oportunidades de los individuos para alcanzar mejores posiciones relativas. Es decir, no resulta claro hasta qué punto esta movilidad social se acompaña de una disminución de la desigualdad de oportunidades en el país.

Torche (2005b) ha documentado, por ejemplo, altos niveles de movilidad ocupacional intergeneracional en Chile, comparables con los de los países industrializados que participan del proyecto CASMIN (Comparative Analysis of Social Mobility in Industrialized Countries). El régimen de estratificación, sin embargo, se caracteriza por fuertes barreras a la movilidad de tipo jerárquico y débiles barreras por sector económico. Es decir, Chile se caracterizaría por altos niveles de movilidad horizontal y bajos niveles de movilidad vertical. Ambas características pueden asociarse claramente al proceso de liberalización radical de la economía chilena, que favorecería la concentración de la riqueza por una parte y la disolución de las barreras sectoriales por la otra. En efecto, considerando únicamente el valor jerárquico -el status relativo- de las ocupaciones, Chile presenta niveles de determinación social del logro individual considerablemente superiores a los de México, o incluso a los que Blau y Duncan (1967) describían para los Estados Unidos de 1962 (Puga y Solís, 2010). Dicho de otra forma, el régimen de movilidad ocupacional chileno es uno de fluidez laboral entre sectores pero de gran desigualdad de oportunidades.

Una situación análoga se produce con la movilidad en términos de ingresos en Chile. Mientras diversos estudios plantean una alta volatilidad de los ingresos, las probabilidades de procesos significativos de movilidad ascendente es comparativamente muy baja, tanto intrageneracional (Contreras et al. 2007) como intergeneracionalmente (Núñez y Risco 2005).

El rol del logro educativo es crucial en el estudio de la transmisión de la desigualdad económica (Shavit y Muller 1998), y por lo tanto resulta esperable que modificaciones 
importantes del sistema educativo, y en particular del sistema educacional público, afecten de modo relevante el régimen de estratificación imperante en una sociedad. En el caso de Chile, la educación es uno de los aspectos que sufrieron grandes transformaciones en el marco de las reformas estructurales. En 1981, el gobierno militar transfirió las escuelas públicas -hasta entonces dependientes del gobierno central- a los municipios, proceso conocido como municipalización de la educación en Chile. Bajo el principio de la descentralización como fórmula de mejoramiento de la eficiencia, y en el contexto de una reducción generalizada del aparato estatal, la educación municipalizada trasladó la gestión educativa a los gobiernos locales y estableció un sistema de subvención fija por alumno para los municipios. La considerable segregación espacial en las grandes ciudades chilenas, así como la concentración de la pobreza en algunas localidades rurales, sin embargo, condicionarían capacidades muy diversas de inversión más allá de la subvención estatal.

En el mismo período se autorizó el financiamiento, por parte del Estado, de escuelas particulares a través de subsidios ${ }^{1}$. Estas escuelas se denominaron particular subvencionadas, y tenían la limitación de no cobrar matrícula. Así, tanto municipios como sostenedores particulares compiten por los fondos públicos de educación. La asignación de recursos se rige teóricamente por las decisiones de las familias, estimulando la competencia entre los oferentes.

El impacto de la municipalización y posteriormente de la educación particular subvencionada en Chile es difícil de menospreciar. Entre 1981 y 1986 se produce una gran expansión de la educación particular subvencionada (del 15.1 al 30.8\% de la matrícula), que se estancaría hasta 1994 tanto por la disminución inicial de la subvención como por la posterior promulgación de leyes que entorpecerían el lucro de estas instituciones. Sin embargo, en 1994 se autoriza el financiamiento compartido -es decir, se autoriza a los sostenedores particulares a complementar la subvención con cobros adicionales-, lo que redunda en nuevo período de expansión, superando estas escuelas el $40 \%$ de la matrícula nacional (Larráñaga, 2004).

Como ha sido establecido en diversos estudios (Rodríguez y Winchester 2001; Sabatini y Wormald 2004; Torche, 2005a), las reformas privatizadoras del sistema educativo han implicado la aparición de nuevas formas de desigualdad educativa al interior de la educación financiada públicamente. Al respecto Torche (2005a) observa que, pese a la expansión general de la cobertura, la transmisión de la desigualdad educativa en Chile no sólo no ha disminuido, sino que tiende a incrementarse en conjunción con las reformas antes referidas.

En el ya descrito marco de baja movilidad jerárquica, el presente trabajo busca explorar cuál es el rol de estas formas de desigualdad educativa en la reproducción de la estratificación social en general, entendiendo en este caso la transmisión del status ocupacional de las personas como la dimensión central en la transmisión intergeneracional

Estrictamente, existen escuelas particulares subvencionadas en Chile desde el siglo XIX, cuando el gobierno de Manuel Montt autorizó el subsidio de escuelas dirigidas por diversas entidades de beneficencia. Desde 1920 la Ley de Instrucción Primaria Obligatoria obligó también a sostener escuelas a los propietarios rurales de ciertas características. Sin embargo, es sólo en el marco de las reformas de 1980 que este sector cobra mayor relevancia, al desregularse fuertemente el sistema educativo y ofrecerse condiciones adecuadas para el desarrollo de lucro privado a partir de la subvención. 
de la posición social y el privilegio en las sociedades modernas. Desde esta perspectiva, cabe preguntarse cuál es el efecto de las reformas al modelo educativo chileno en la movilidad ocupacional de las personas. Es decir, este trabajo explora el potencial vínculo entre las políticas de educación pública y la reproducción de la desigualdad social entre las personas una vez que abandonan el sistema formal de enseñanza.

La pregunta es particularmente pertinente atendiendo a la polémica que la desigualdad educativa produce en un país de alta desigualdad como es Chile. En efecto, la desigualdad en las oportunidades educativas es por mucho el aspecto de la desigualdad social frente al que la población en el país toma posiciones más críticas (Puga, 2011). El carácter conflictivo de la desigualdad educativa y sus efectos sobre la reproducción de la desigualdad en general se refleja en el alto impacto de las movilizaciones estudiantiles en la última década en Chile. Se trata, en definitiva, de una dimensión de la desigualdad social que violenta particularmente la percepción de justicia en la sociedad chilena.

La significación del problema puede expandirse más aún si se consideran las argumentaciones de carácter técnico-pedagógico y político-normativas involucradas en la discusión sobre la reforma, privatización y descentralización de la educación en Chile. En términos generales, la municipalización y el estímulo a la educación particular subvencionada se sustenta en la creencia en la menor efectividad de la administración estatal del proceso educativo, que redundaría en peores resultados para quienes acceden a la educación pública. El sistema de subsidios al estudiante -en lugar de a la escuela- que caracteriza la educación particular subvencionada, por otro lado, se sustenta en el principio de libertad de enseñanza, que identifica como negativo el proceso de homogenización asociado a la administración centralizada de la educación, y como valor positivo que el Estado subsidie una diversidad de posibles proyectos educativos entre los que los hogares pueden escoger.

Respecto de las argumentaciones técnico pedagógicas, existe cada vez mayor consenso sobre la escasa diferencia en efectividad entre las escuelas particular subvencionadas y las escuelas públicas, una vez controlados los efectos del nivel socioeconómico de los estudiantes y sus hogares. Castillo et al. (2011), a partir del análisis de los resultados del sistema de evaluación de la educación SIMCE, concluyen que frente a estudiantes en igualdad de condiciones socioeconómicas las escuelas públicas se desempeñan académicamente igual o mejor que las escuelas subvencionadas, particularmente en los segmentos de menores recursos. Todo esto, inclusive, cuando es imposible controlar adecuadamente el efecto de las prácticas de selección y de los recursos adicionales con que cuentan las escuelas particulares.

Esto no implica, sin embargo, que la modalidad escolar no tenga efectos sobre las posibilidades de movilidad social de las personas más allá de su extracción social. Aún cuando las escuelas de diverso tipo tengan una efectividad pedagógica similar, el logro educativo y ocupacional puede verse afectado por diversas variables asociadas al entorno cuando se establece un sistema educativo socialmente segregado: reducción de expectativas, discriminación, redes de contacto diferenciadas, etc. Es decir, es posible que, aun cuando la educación particular subvencionada no represente ninguna ventaja para el sistema educativo en términos educativos, sí represente un mecanismo adicional para la reproducción de las desigualdades sociales que afectan al país. El rol de la escuela en la estratificación social, como espacio de aprendizaje formal pero 
también de socialización, va mucho más allá de la generación de la cualificación de los educandos.

Comprender el efecto de diversas modalidades educativas en la transmisión intergeneracional de la posición social involucra considerar dos grandes momentos en el proceso de estratificación. Por una parte, la determinación de la modalidad educativa del individuo a partir de la situación social de su hogar de origen. En segundo lugar, la determinación del logro educativo del individuo a partir de la modalidad educativa tomada, junto a las características de su origen social. Por la otra, la determinación de la posición social del individuo a partir de la modalidad educativa, su logro educativo, y las características de su origen social. Es decir, es necesario distinguir efectos directos e indirectos de la modalidad educativa sobre el proceso de logro ocupacional, y comprender a su vez a la modalidad educativa como variable intermedia en la transmisión de la posición social entre dos generaciones.

Atendiendo estas consideraciones, presento un análisis del papel de las modalidades educativas en la reproducción de la desigualdad social en Santiago de Chile. Restringir el análisis a la población de la ciudad capital implica controlar diversos aspectos, particularmente las limitaciones al logro ocupacional dadas por condiciones de aislamiento y ruralidad -los llamados efectos sectoriales de la movilidad (Erikson y Goldthorpe 1992)-, aunque no necesariamente las limitaciones similares al logro educativo -al no excluirse la población migrada-. Esto permite concentrar el análisis en el impacto de las variables educativas en el logro.

\section{DATOS Y TÉCNICAS}

El análisis siguiente se basa en la información proveniente de la EMSC (Encuesta de Movilidad Social en Chile), realizada el año 2001 por Florencia Torche, Guillermo Wormald y la Dirección de Estudios Sociológicos de la Pontificia Universidad Católica de Chile. La encuesta posee una muestra total de 3.544 casos, probabilística, de nivel nacional, en la que se seleccionó a hogares con jefes varones de entre 24 y 69 años. La muestra efectivamente utilizada, correspondiente a la población residente en Santiago de Chile con al menos un año de escolaridad formal en Chile, es de 1.736 casos.

Con estos datos, se utilizan técnicas de regresión lineal múltiple (OLS) para modelar la determinación del logro educativo y ocupacional (actual e inicial) de los entrevistados, entendiendo al primero como factor central en este último.

El rango de edad de los entrevistados permite comparar casos que asistieron tanto al modelo público estatal de educación como al modelo público municipalizado, así como individuos que asistieron a escuelas particulares subvencionadas o privadas. Al mismo tiempo, el amplio rango de edad afecta los resultados en términos de logro ocupacional en dos sentidos: (1) el logro ocupacional tiende a incrementarse con el tiempo individual, en la medida en que la carrera laboral se consolida, y (2) el logro ocupacional se ve afectado por la cohorte de los individuos (tiempo histórico), dadas las transformaciones de la estructura productiva y laboral. Por estas razones, se ha modelado el impacto de las modalidades escolares controlando el efecto del momento de nacimiento de los individuos, lo que permitiría aislar los efectos sincrónicos que se busca determinar. Al respecto, cabe destacar que se ensayaron diversas combinaciones de clasificación de cohortes para ser 
utilizadas en conjunto con la edad como variables de control, con el fin de captar por un lado la variación lineal asociada al tiempo individual (edad) y la variación posiblemente no lineal asociada al tiempo histórico (cohortes). Sin embargo, en los diversos modelos las variables de cohorte no captaron mayor capacidad explicativa que la ya incluida en el efecto lineal de la edad, ni afectaron los coeficientes de las variables en estudio. Por esto, se conservó sólo el efecto lineal de tiempo en pos de un modelo más parsimonioso.

Como medida del logro ocupacional -tanto del entrevistado como del padre- se utilizó el ISEI (International Socio-Economic Index of Occupational Status) asociado a cada una de las ocupaciones (clasificadas de acuerdo a la ISCO88) de acuerdo a la propuesta de Ganzeboom y Treiman (1996). El ISEI busca ser una medida de validez internacional del status social y económico representado por diversas ocupaciones, y está expresado en una escala que varía entre 16 (mínimo) y 90 (máximo status).

La modalidad educativa fue capturada en la EMSC a través de dos preguntas respecto de la modalidad de estudios cursada para la educación primaria y secundaria. Respecto de esta clasificación es necesario realizar algunas aclaraciones. Se ensayaron dos formas de clasificación, una basada únicamente en la educación primaria, y otra utilizando la forma de educación secundaria como criterio primario y la educación primaria sólo cuando no existió educación secundaria. Al comparar los modelos utilizando ambos criterios, se obtiene que resulta más adecuado utilizar como indicador la educación primaria. Esto resulta concordante con diversos estudios que reportan la relevancia crucial de los primeros años de instrucción en la determinación del logro de los individuos.

Los individuos tienden a permanecer en el mismo régimen educativo en ambas etapas escolares, aunque se producen niveles considerables de transición entre modalidades educativas (ver Tabla 1). Esto es particularmente notorio para la distinción entre educación pública estatal y educación pública municipalizada, lo que resulta esperable al existir una generación que necesariamente debió mudar de una a otra al realizarse el proceso de municipalización.

Respecto de la clasificación de las modalidades educativas al interior de cada etapa escolar, la distinción entre educación pública estatal y educación pública municipalizada no se produce a través del mismo cuestionario sino que se infiere a partir de la edad de los individuos. La municipalización de la educación pública se produce de forma universal en 1981, por lo que la distinción entre ambas formas de educación pública se traslapa necesariamente con la cohorte de los individuos. Cierto nivel de error puede producirse en esta distinción al no contar la EMSC con información sobre cuándo exactamente realizan sus estudios las personas, pero éste debiera ser marginal, especialmente al utilizarse la educación primaria como criterio de clasificación.

Comprender el efecto de la modalidad educativa tanto en la escolaridad de los individuos como en los inicios de la vida laboral, así como el efecto de estos dos elementos en el logro ocupacional de las personas, permite comprender a cabalidad los efectos en el tiempo de la segregación social de la educación. Para estos efectos, una formulación especialmente adecuada es la de modelos de adquisición de status utilizando path analysis. Lamentablemente, sin embargo, el carácter cualitativo y polinomial de la distinción entre diversas modalidades educativas dificulta su inclusión como variable endógena en estos modelos. 


\section{Tabla 1}

Modalidad Educativa en Educación Secundaria según Modalidad en Educación Primaria

\begin{tabular}{|l|c|r|r|r|}
\hline \multicolumn{5}{|c|}{ Personas con al menos un año de educación primaria } \\
\hline Educación Secundaria & \multicolumn{4}{|c|}{ Educación primaria } \\
\hline Municipalizada & Municipalizada & Estatal & Part. Subv. & Privada \\
\hline Estatal & $48.9 \%$ & $7.8 \%$ & $7.1 \%$ & $5.2 \%$ \\
\hline Part. Subvencionada & $0.0 \%$ & $40.3 \%$ & $24.3 \%$ & $17.1 \%$ \\
\hline Particular Privada & $17.9 \%$ & $7.9 \%$ & $51.3 \%$ & $7.2 \%$ \\
\hline No Aplica & $5.9 \%$ & $3.1 \%$ & $4.5 \%$ & $65.4 \%$ \\
\hline $\mathrm{n}$ & $27.2 \%$ & $41.0 \%$ & $12.7 \%$ & $5.1 \%$ \\
\hline \multicolumn{5}{|c|}{ Personas con al menos un año de educación secundaria } \\
\hline & 243 & 1102 & 170 & 221 \\
\hline Educación Secundaria & Eunicipalizada & Estatal & Part. Subv. & Privada \\
\hline Municipalizada & $67.2 \%$ & $13.2 \%$ & $8.2 \%$ & $5.5 \%$ \\
\hline Estatal & $0.0 \%$ & $68.2 \%$ & $27.8 \%$ & $18.0 \%$ \\
\hline Part. Subvencionada & $24.7 \%$ & $13.4 \%$ & $58.8 \%$ & $7.6 \%$ \\
\hline Particular Privada & $8.1 \%$ & $5.2 \%$ & $5.2 \%$ & $68.9 \%$ \\
\hline $\mathrm{n}$ & 179 & 653 & 150 & 208 \\
\hline
\end{tabular}

\section{RESULTADOS}

Un primer paso al describir el papel de la diferenciación por modalidades escolares al interior de la educación financiada por el Estado sobre la estratificación social es comprender cómo se asocia la posición social de los hogares con el tipo de educación recibida por los entrevistados. En este aspecto, la distinción entre escuelas públicas estatales y municipalizadas resulta espuria, ya que desde la perspectiva de los hogares ambas modalidades ocupan el mismo rol -educación pública gratuita con restricciones a la aplicación de criterios de selección-, y no es posible optar entre ambas en ningún momento del tiempo. En efecto, al momento de la reforma las escuelas municipalizadas son las mismas escuelas estatales que cambian de administración y estatuto.

Como se observa en la Tabla 2, más de tres cuartas partes de los entrevistados realizó su educación primaria en la educación pública. Esta proporción cambia radicalmente, sin embargo, de acuerdo a la posición social del padre. En efecto, el $92.7 \%$ de los entrevistados cuyos padres están en el $20 \%$ inferior de la distribución por ISEI (16/22) asistió a una escuela pública, mientras que sólo lo hizo el $26.0 \%$ de quienes cuyos padres están en el $5 \%$ superior de esta misma distribución (63/88). La educación particular subvencionada 
Tabla 2

Status ocupacional del padre (ISEI) y modalidad escolar en educación primaria

\begin{tabular}{|l|c|c|c|c|}
\hline \multicolumn{5}{|c|}{ Personas con al menos un año de educación primaria } \\
\hline & \multicolumn{4}{|c|}{ Educación Primaria } \\
\hline iSEI del padre & Mun./Estatal & Part. Subv. & Privada & $\mathrm{n}$ \\
\hline $16 / 22$ & $92.7 \%$ & $4.3 \%$ & $3.1 \%$ & 258 \\
\hline $23 / 28$ & $83.5 \%$ & $8.3 \%$ & $8.2 \%$ & 241 \\
\hline $29 / 32$ & $82.1 \%$ & $10.6 \%$ & $7.3 \%$ & 358 \\
\hline $33 / 41$ & $75.9 \%$ & $11.3 \%$ & $12.9 \%$ & 319 \\
\hline $42 / 52$ & $66.1 \%$ & $12.6 \%$ & $21.2 \%$ & 171 \\
\hline $53 / 62$ & $41.8 \%$ & $16.5 \%$ & $41.8 \%$ & 63 \\
\hline $63 / 88$ & $26.0 \%$ & $12.9 \%$ & $61.2 \%$ & 70 \\
\hline Total & $76.5 \%$ & $9.8 \%$ & $13.7 \%$ & 1480 \\
\hline
\end{tabular}

caracteriza claramente a los sectores medios-altos, siendo la educación particular privada claramente preponderante en el 20\% superior de la distribución (42/52).

Lejos de atenuarse al desarrollarse el mecanismo de subvenciones estatales a la educación privada, la segregación social entre distintas modalidades escolares se ha incrementado a través del tiempo. Como puede observarse en los análisis de varianza de la Tabla 3, la asociación entre ISEI del padre y modalidad escolar se incrementa marcadamente en las cohortes más jóvenes.

\section{Tabla 3}

Análisis de varianza: ISEI del padre y modalidad escolar en educación primaria por cohorte

\begin{tabular}{|l|c|c|}
\hline \multicolumn{3}{|c|}{$\begin{array}{c}\text { Personas con al menos un año } \\
\text { de educación primaria }\end{array}$} \\
\hline Coef. F & Cohorte & $\mathrm{n}$ \\
\hline $1937 / 1944$ & $1.75^{* *}$ & 258 \\
\hline $1945 / 195$ & $22.18^{* * *}$ & 241 \\
\hline $1953 / 1960$ & $2.55^{* * *}$ & 358 \\
\hline $1961 / 1968$ & $4.27 * * *$ & 319 \\
\hline $1969 / 1976$ & $4.51^{* * *}$ & 202 \\
\hline$* \mathrm{p}<=.10^{* *}$ & $\mathrm{p}<=.05$ & $* * * \mathrm{p}<=.01$ \\
\hline
\end{tabular}


Como se reportó anteriormente, existe evidencia de que las escuelas particulares subvencionadas no obtienen mejores resultados pedagógicos que las escuelas públicas una vez controlado el efecto de los orígenes sociales de los estudiantes. En efecto, más bien las mismas escuelas particulares subvencionadas se caracterizarían por entregar una educación segmentada y desigual según el status de sus estudiantes (Castillo et al. 2011). Sin embargo, es posible hipotetizar que el mismo efecto de segregación social en el proceso educativo tiende a reproducir la desigualdad social aún cuando la gestión educacional privada no sea superior a la pública.

Para evaluar estos posibles efectos, se modela el impacto de la modalidad escolar en la educación primaria de los entrevistados sobre su logro ocupacional en el año 2001. Se presentan tres modelos progresivos para mejor describir el posible impacto de la modalidad educativa.

En un primer modelo, el logro ocupacional -operacionalizado como ISEI- es determinado únicamente por el año de nacimiento del entrevistado (efectos edad y cohorte) y la modalidad escolar. En el segundo modelo se ingresa como variable de control el nivel de escolaridad alcanzado por los individuos, y en el tercero se ingresan las variables asociadas al hogar de origen: ISEI del padre a los 14 años del entrevistado, y escolaridad del padre. En un principio la escolaridad de la madre fue incluida también en este tercer modelo, pero el efecto no resultó significativo y la falta de información reducía innecesariamente el número de casos en el análisis.

Como puede observarse en el Cuadro 1, todos los coeficientes indican efectos significativos en el primer modelo. El año de nacimiento posee un efecto positivo y significativo, lo que implica que los entrevistados de las cohortes más jóvenes alcanzan posiciones ocupacionales más altas. Esto es concordante con la transformación económica del país en los últimos 50 años, y se encuentra probablemente subestimado gracias al efecto inverso de la edad de los entrevistados sobre su posición ocupacional.

La modalidad de educación municipalizada actúa como referencia en todos los modelos. Podemos observar en el modelo 1 que la educación estatal es la más similar en su impacto sobre el futuro logro ocupacional, aunque posee un efecto positivo con significación superior al 95\%. Esto implica que, antes de la municipalización, los estudiantes de la educación pública tendían a obtener posiciones ocupacionales relativamente superiores: es decir, efectivamente la municipalización ha afectado negativamente las oportunidades de los estudiantes de las escuelas públicas en general.

Como resulta esperable, el efecto de estudiar en una escuela privada es muy importante y claramente significativo, aumentando fuertemente el logro ocupacional de los entrevistados. El efecto de estudiar en una escuela particular subvencionada es también claramente significativo, y muy superior al de estudiar en una escuela estatal. Es decir, efectivamente la distinción entre ambas escuelas aportaría una mayor diferenciación en términos de logro ocupacional, que gracias a los resultados presentados anteriormente podemos identificar como conducentes a la reproducción de las desigualdades sociales, y crecientes en el tiempo.

Pasando al análisis del modelo 2, observamos que el impacto de las diversas modalidades educativas decrece de modo importante al incorporarse información sobre la escolaridad de los individuos. Esto sugeriría que, en realidad, parte importante del efecto observado primero se produce por diferencias en la escolaridad final de los estudiantes en las distintas modalidades. Únicamente las escuelas particulares privadas conservan un 


\section{Cuadro 1}

Determinación del status ocupacional

\begin{tabular}{|c|c|c|c|}
\hline \multicolumn{4}{|c|}{$\begin{array}{l}\text { Modelo de regresión lineal múltiple (OLS) } \\
\text { Coeficientes de regresión estandarizados }\end{array}$} \\
\hline Variable/Modelo & Mod 1 & Mod 2 & Mod 3 \\
\hline Año de nacimiento & $0.088 * * *$ & $-0.113 * * *$ & $111 * * *$ \\
\hline \multicolumn{4}{|l|}{ Modalidad de escuela (prim) } \\
\hline Municipalizada (ref) & - & - & - \\
\hline Estatal & $0.094 * *$ & 0.031 & 0.008 \\
\hline Part. Subvencionada & $0.164 * * *$ & 0.035 & 0.024 \\
\hline Particular Privada & $0.444 * * *$ & $0.147 * * *$ & $0.082 * *$ \\
\hline Escolaridad (años) & & $0.642 * * *$ & $0.545^{* * *}$ \\
\hline \multicolumn{4}{|l|}{ Orígenes sociales } \\
\hline Logro ocupacional padre & & & $0.153^{* * *}$ \\
\hline Escolaridad del padre & & & $0.070 * *$ \\
\hline \multicolumn{4}{|l|}{ Coeficientes de ajuste } \\
\hline Coef. F & $79.83 * * *$ & $295.23^{* * *}$ & $171.71 * * *$ \\
\hline R2 & $0.160-$ & $0.468-$ & $0.507-$ \\
\hline R2 ajustado & $0.158-$ & $0.466-$ & $0.504-$ \\
\hline $\mathrm{N}$ & $1.687-$ & $1.686-$ & 1.179 \\
\hline$* \mathrm{p}<=.10^{* *} \quad \mathrm{p}<=.05^{* * *}$ & $=.01$ & & \\
\hline
\end{tabular}

efecto importante y significativo, que sugeriría un incremento en las oportunidades más allá de la mayor posibilidad de acceder a una alta escolaridad de sus estudiantes.

Otro aspecto interesante del modelo 2 es el cambio de signo del efecto de cohorte. Este indica que, una vez controlado el efecto producido por el aumento generalizado de la escolaridad, el ISEI de los individuos decae con el tiempo.

Al ingresar las variables asociadas al hogar de origen, en el modelo 3 las diferencias entre modalidades escolares decrecen aún más. El efecto de las escuelas particulares subvencionadas, así como de las escuelas estatales, se acerca claramente al cero. El efecto de la escolaridad también se reduce, lo que responde a la correlación entre la posición social de origen y la escolaridad alcanzada. Los efectos de la posición y escolaridad del padre son, como se esperaba, muy significativos.

Estos resultados indicarían que, una vez controlada la escolaridad de los individuos, la modalidad administrativa de la escuela no tendría efecto en la posición ocupacional de los habitantes de Santiago de Chile, y mucho menos una vez controlados los efectos de la posición social de origen. 
Sin embargo, los efectos teóricamente esperables de la creciente diferenciación entre modalidades escolares sobre el logro ocupacional de los individuos no son necesariamente directos. Particularmente cuando consideramos los efectos adicionales al resultado de una distribución segregada de los estudiantes por modalidad, nos encontramos con mecanismos que operarían primariamente a través de la escolaridad y del ingreso al mundo del trabajo. Para explorar estos efectos indirectos es necesario considerar el impacto de las modalidades de escuela en estas variables intervinientes.

Figura 1

El proceso de estratificación social, Blau y Duncan (1967)

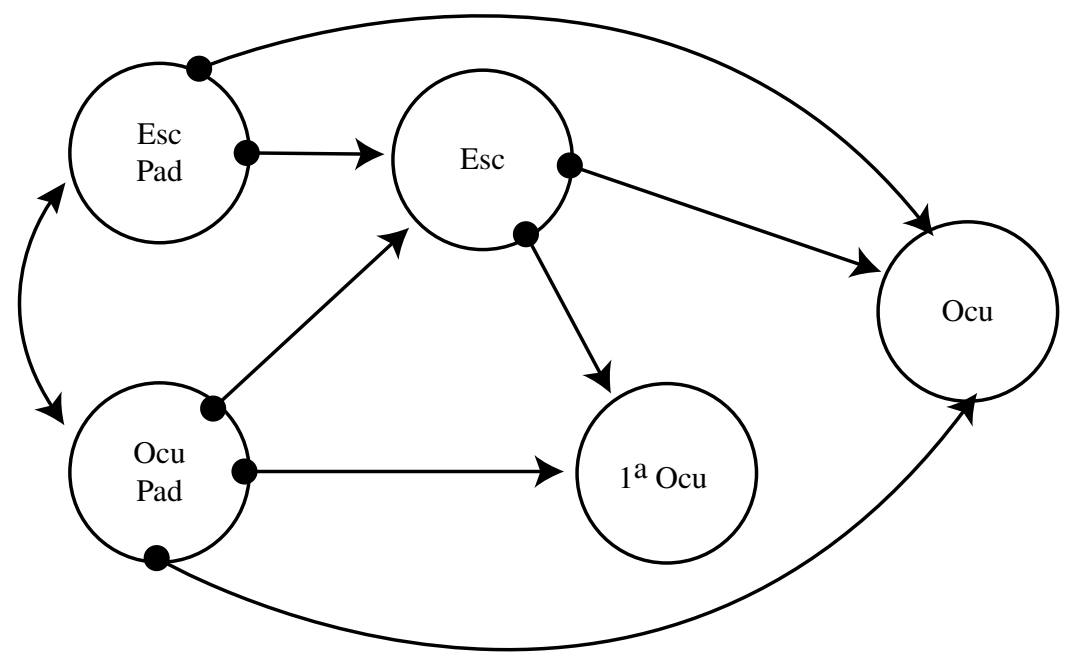

En 1967, Blau y Duncan inauguraron la perspectiva de la "adquisición de status" al proponer el modelo graficado en la Figura 1 para comprender el proceso de estratificación social. Modificando este modelo, es posible proponer una alternativa para evaluar el efecto real de la municipalización y privatización de la educación pública en la transmisión de las desigualdades en Chile. En este modelo se incorpora el efecto de la escolaridad de la madre sobre la del individuo, que ha sido reportada como relevante previamente para el caso de Chile (Torche, 2005a), y la mediación de la modalidad escolar sobre el logro educativo y la integración al mundo laboral.

Si bien es difícil explorar en un path analysis la determinación de una variable polinomial como la modalidad escolar, sí es posible determinar los efectos sobre la escolaridad, la primera ocupación y la ocupación actual de los individuos, considerando las directrices del modelo para determinar el efecto total de las modalidades de escuela sobre la posición social de los individuos, una vez controlados los efectos directos de la posición social de origen, la escolaridad y la primera ocupación. 
Figura 2

Modelo modificado de estratificación social

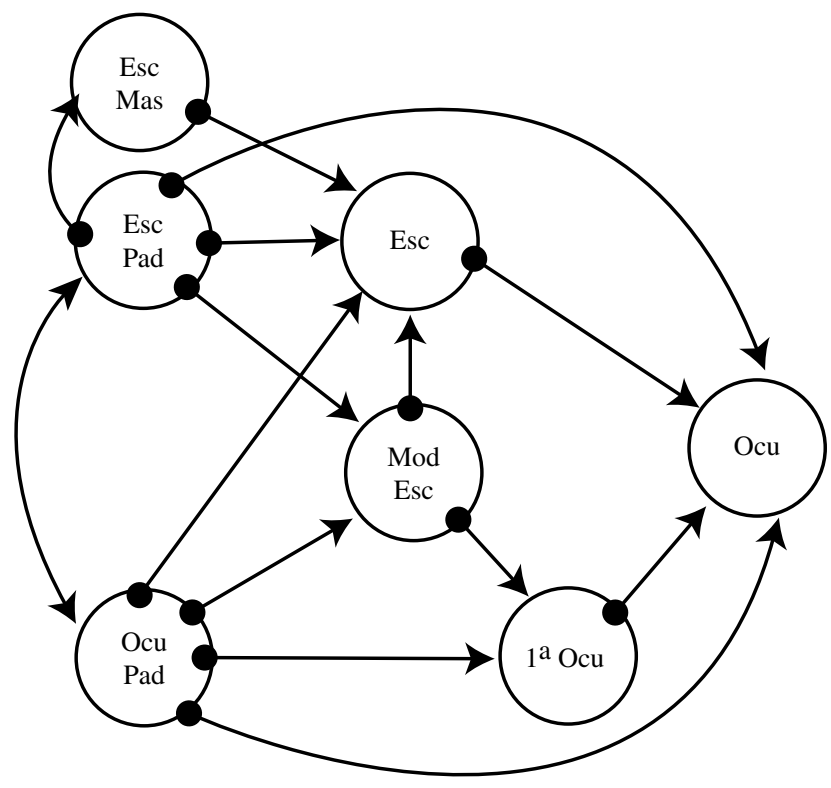

Los modelos reportados en el Cuadro 2 exploran los efectos de las distintas modalidades de administración escolar sobre la escolaridad de los individuos. En el primer modelo se calculan los efectos únicamente de la modalidad escolar y el año de nacimiento. Como es esperable dada la masiva expansión de la escolaridad en Chile, existe un fuerte efecto positivo del año de nacimiento. Por otro lado, todas las modalidades de escuela poseen un efecto positivo fuerte y significativo $(a<0.01)$ en relación a la educación municipalizada, que opera como referencia.

En el modelo 2 ingresan como variables de control el status ocupacional y la escolaridad del padre, así como la escolaridad de la madre. Esta última variable sí presenta un claro efecto significativo en este caso, superior de hecho al de la escolaridad del padre.

Resulta crucial observar que, en este segundo modelo, el efecto de las distintas modalidades de escuela sigue siendo significativo tras ingresar las variables de origen social. Estos efectos no pueden ya atribuirse al fenómeno de selección operante en cada una de las modalidades. Es decir, el proceso de municipalización y la expansión de la educación particular subvencionada efectivamente aportan a la mayor transmisión de las desigualdades educativas. El efecto de estudiar en una escuela estatal versus una municipalizada ( $a=0.055$ ) indicaría que los estudiantes de escuelas públicas tienen, aún tras controlar el efecto de cohorte y de origen social, menores oportunidades de logro educativo a partir de la municipalización de las escuelas. Más fuerte y significativo es el impacto de estudiar en una escuela particular subvencionada, lo que implica que la 


\section{Cuadro 2}

Determinación de la escolaridad

\begin{tabular}{|c|c|c|}
\hline \multicolumn{3}{|c|}{$\begin{array}{l}\text { Modelo de regresión lineal múltiple (OLS) } \\
\text { Coeficientes de regresión estandarizados }\end{array}$} \\
\hline Variable/Modelo & Mod 1 & Mod 2 \\
\hline Año de nacimiento & $0.311 * * *$ & $0.189 * * *$ \\
\hline \multicolumn{3}{|l|}{ Modalidad de escuela (prim) } \\
\hline Municipalizada (ref) & - & - \\
\hline Estatal & $0.096 * * *$ & $0.083 *$ \\
\hline Part. Subvencionada & $0.210 * * *$ & $0.120 * * *$ \\
\hline Particular Privada & $0.457 * * *$ & $0.242 * * *$ \\
\hline \multicolumn{3}{|l|}{ Origenes sociales } \\
\hline Logro ocupacional padre & & $0.177 * * *$ \\
\hline Escolaridad del padre & & $0.150 * * *$ \\
\hline Escolaridad de la madre & & $0.268 * * *$ \\
\hline \multicolumn{3}{|l|}{ Coeficientes de ajuste } \\
\hline Coef. F & $142.2 * * *$ & $111.11 * * *$ \\
\hline $\mathrm{R}^{2}$ & $0.247-$ & 0.459 \\
\hline R2 ajustado & $0.246-$ & $0.455-$ \\
\hline $\mathrm{N}$ & $1735-$ & $926-$ \\
\hline$* * \mathrm{P}<=.05$ & $<=.01$ & \\
\hline
\end{tabular}

creciente segmentación de la educación financiada públicamente apunta hacia la generación de una estructura cada vez más estratificada de oportunidades educativas, adicionalmente a la desigualdad de oportunidades que implica una sociedad con indicadores distributivos como los de Chile.

Los modelos presentados en el Cuadro 3 exploran los efectos de la modalidad escolar en el status ocupacional inicial de los individuos. Es decir, de su posición social al integrarse al mercado del trabajo. Siguiendo el procedimiento antes utilizado, en el primer modelo se incluyen únicamente el año de nacimiento y la modalidad escolar como variables independientes. Los resultados indican que existe claramente un efecto significativo de la modalidad escolar en la integración laboral de las personas.

Al ingresar la escolaridad del entrevistado y el status ocupacional del padre como variables de control (siguiendo el modelo expuesto en la Figura 2), ocurren dos cambios centrales. Por una parte, el año de nacimiento pasa a tener un efecto negativo, igualmente significativo. Ya que estamos controlando por escolaridad, esto probablemente implica que, a igual escolaridad, el status ocupacional inicial de las personas ha ido disminuyendo en el tiempo junto con la expansión generalizada de la educación formal en el país. Por 
otra parte, la diferencia entre escuelas municipalizadas y escuelas estatales pierde significación. A modo de conjetura, podríamos proponer que la diferencia en la ocupación inicial producida por la modalidad escolar -ya controladas las variables de escolaridad y origen social- opera principalmente a través de mecanismos como la discriminación positiva y negativa de los empleadores, lo que explicaría que la diferencia entre escuelas municipalizadas y estatales se disipe. En términos simbólicos (la escuela pública), el status de las escuelas estatales y municipalizadas es bastante similar.

\section{Cuadro 3}

Determinación del status ocupacional inicial

\begin{tabular}{|c|c|c|}
\hline \multicolumn{3}{|c|}{$\begin{array}{l}\text { Modelo de regresión lineal múltiple (OLS) } \\
\text { Coeficientes de regresión estandarizados }\end{array}$} \\
\hline Variable/Modelo & Mod 1 & Mod 2 \\
\hline Año de nacimiento & $0,094 * * *$ & $-0,085^{* * *}$ \\
\hline \multicolumn{3}{|l|}{ Modalidad de escuela (prim) } \\
\hline Municipalizada (ref) & - & - \\
\hline Estatal & $0,123 * * *$ & 0,052 \\
\hline Part. Subvencionada & $0,210 * * *$ & $0,077 * * *$ \\
\hline Particular Privada & $0,440 * * *$ & $0,113^{* * *}$ \\
\hline Escolaridad (años) & & $0,506^{* * *}$ \\
\hline \multicolumn{3}{|l|}{ Orígenes sociales } \\
\hline Logro ocupacional padre & $0,215 * * *$ & \\
\hline \multicolumn{3}{|l|}{ Coeficientes de ajuste } \\
\hline Coef. F & $75,69 * * *$ & $205,75^{* * *}$ \\
\hline $\mathrm{R} 2$ & $0,151-$ & 0,460 \\
\hline R2 ajustado & $0,149-$ & $0,457-$ \\
\hline $\mathrm{N}$ & $1706-$ & 1459 \\
\hline$* * \mathrm{P}<=.05$ & $=.01$ & \\
\hline
\end{tabular}

Lo central, sin embargo, es que el efecto de estudiar en una escuela particular subvencionada sigue siendo muy significativo. El efecto de la diferenciación interna de la educación financiada públicamente, entonces, nuevamente produce efectos que van más allá del efecto de selección de los estudiantes. Nuevamente como hipótesis, podemos proponer que la diferenciación en la posición inicial de los egresados de escuelas públicas y particular subvencionadas persiste porque (a) la diferenciación simbólica entre ambas modalidades sí es efectiva, permitiendo la discriminación por parte los empleadores, y/o (b) porque la segregación social de los estudiantes genera diferencias en términos de capital social y redes en general, lo que modifica las posibilidades individuales de 
integración. Si consideramos el impacto que la posición ocupacional inicial puede tener en el logro de los trabajadores en el tiempo, la hipótesis de que la diversificación de las modalidades escolares ha conducido a una mayor estratificación cobra aún más fuerza.

Finalmente, queda considerar los efectos directos de la modalidad escolar en el logro ocupacional de las personas una vez consideradas todas las variables del modelo ya expuesto en la Figura 2. Éstas corresponden a la escolaridad de ego, la ocupación inicial, y la escolaridad del padre. Como variable de control anexa por las condiciones de la muestra, se incluye nuevamente el tiempo histórico (el año de nacimiento).

\section{Cuadro 4}

Determinación del status ocupacional considerando ocupación inicial y escolaridad de los individuos

\begin{tabular}{|c|c|}
\hline \multicolumn{2}{|c|}{$\begin{array}{l}\text { Modelo de regresión lineal múltiple (OLS) } \\
\text { Coeficientes de regresión estandarizados }\end{array}$} \\
\hline Variable/Modelo & Mod 1 \\
\hline Año de nacimiento & $-0,065 * * *$ \\
\hline \multicolumn{2}{|l|}{ Modalidad de escuela (prim) } \\
\hline Municipalizada (ref) & - \\
\hline Estatal & $-0,001$ \\
\hline Part. Subvencionada & $-0,002$ \\
\hline Particular Privada & 0,050 \\
\hline Escolaridad (años) & $0,358 * * *$ \\
\hline \multicolumn{2}{|l|}{ Orígenes sociales } \\
\hline Logro ocupacional padre & $0,097 * * *$ \\
\hline Escolaridad del padre & $0,053^{*}$ \\
\hline \multicolumn{2}{|l|}{ Coeficientes de ajuste } \\
\hline Coef. F & $197,53^{* * *}$ \\
\hline $\mathrm{R} 2$ & $0,577-$ \\
\hline R2 ajustado & $0,574-$ \\
\hline $\mathrm{n}$ & $1166-$ \\
\hline$* \mathrm{p}<=.10 \quad * * \mathrm{p}<=.05$ & .01 \\
\hline
\end{tabular}

Como se esperaba dados los resultados expuestos en el Cuadro 1, en estas condiciones la modalidad escolar no muestra un efecto directo significativo sobre las posiciones ocupacionales de los individuos (Cuadro 4). Esto es efectivo incluso para la educación particular pagada, lo que deja en evidencia que el hecho de que tales efectos sean únicamente indirectos no implica en ningún caso que resulten menos relevantes. El R2 del 
modelo, de .577 , nos indica que el logro ocupacional de los individuos está fuertemente determinado por los orígenes sociales, por la ocupación inicial, y por la escolaridad de las personas. En todas estas variables la modalidad escolar posee un efecto diferenciador significativo que resulta necesario considerar.

Vale entonces la pena realizar el cálculo de los efectos indirectos de cada una de las modalidades educativas en comparación con la educación municipalizada, considerando la mediación de la escolaridad y del ingreso al mundo del trabajo. Sólo de esta forma es posible apreciar adecuadamente el impacto de la reforma educacional en el régimen de estratificación.

Podemos modelar el efecto de la modalidad escolar a través de un segmento del modelo modificado de estratificación social presentado en la Figura 2. Para realizar estos cálculos, se ha considerado que los efectos no significativos tendrán un valor de cero, con el fin de evitar inferencias espurias. En este modelo parcial presentado en la Figura 3, no resulta relevante la determinación de variables como la escolaridad del individuo ni la modalidad escolar, y se han retirado los efectos directos de la modalidad escolar sobre el logro ocupacional en base a los resultados anteriores.

\section{Figura 3}

Modelación del efecto total de la modalidad escolar

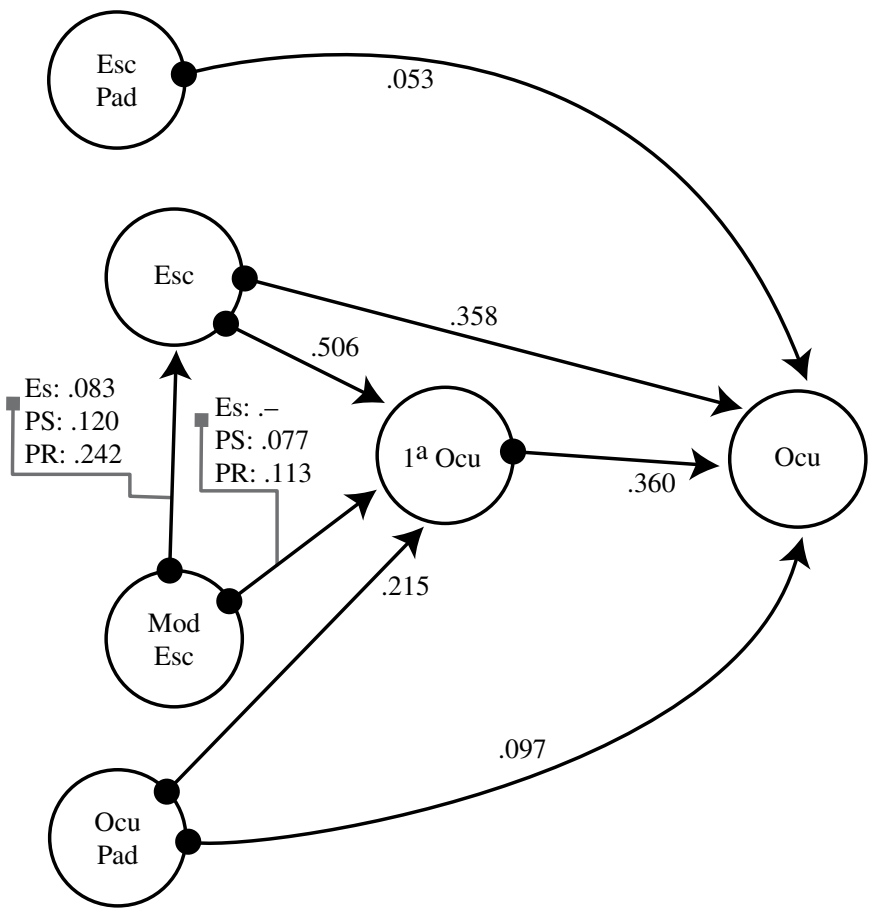


Del modelo, se obtiene que el efecto de la modalidad escolar sobre la posición ocupacional de las personas equivale a su efecto sobre la escolaridad multiplicado por el efecto de la escolaridad sobre el logro final, sumado al efecto de la modalidad sobre la posición inicial multiplicado por el efecto de esta posición sobre la posición ocupacional actual. Siendo la modalidad escolar una variable categórica y polinomial, estos efectos sólo pueden calcularse como los efectos acumulados de haber estudiado en uno u otro tipo de escuela en comparación a una categoría de referencia, en este caso las escuelas municipalizadas. Estos efectos se resumen en la Tabla 4.

\section{Tabla 4}

Efectos de la modalidad escolar sobre la posición ocupacional, controlados los efectos del origen social

\begin{tabular}{|l|c|c|c|}
\hline Modalidad Escolar & $\begin{array}{c}\text { Efecto Directo } \\
\text { sobre la Escolaridad }\end{array}$ & $\begin{array}{c}\text { Efecto Directo sobre } \\
\text { la Ocupación Inicial }\end{array}$ & $\begin{array}{c}\text { Efecto Indirecto sobre la } \\
\text { Posición Ocupacional }\end{array}$ \\
\hline Municipalizada (ref) & - & - & - \\
\hline Estatal & 0,083 & - & 0,030 \\
\hline Part. Subvencionada & 0,120 & 0,077 & 0,071 \\
\hline Particular Privada & 0,242 & 0,113 & 0,127 \\
\hline$* \mathrm{P}<=-10 \quad * * \mathrm{P}<=.05$ & $* * * \mathrm{P}<=.01$ \\
\hline
\end{tabular}

De esta forma, el efecto negativo acumulado sobre las perspectivas de logro ocupacional por haber estudiado en una escuela municipalizada frente a una escuela pública estatal es de un $3 \%$. El efecto positivo de estudiar en una escuela particular subvencionada es de poco más que un 7\%, mientras que el de estudiar en una escuela particular privada se acerca al $13 \%$. Si estos números por sí solos parecen modestos, debe considerarse que se trata de efectos adicionales a los que la posición social de los padres genera. Dicho de otro modo, se trata de efectos que vienen a incrementar el ya documentado fenómeno de transmisión de la desigualdad en Chile.

\section{CONCLUSIONES}

Los resultados anteriores pueden resumirse en tres grandes conclusiones de relevancia sobre el efecto de las reformas educativas en las perspectivas de logro de la población de Santiago de Chile.

En primer lugar, la modalidad de la escuela donde se estudia no posee efectos directos sobre el logro ocupacional de los individuos. Esto es concordante con la investigación existente sobre la efectividad escolar de las instituciones públicas y particular subvencionadas en Chile (Castillo et al. 2011) en el sentido de que, dada una posición inicial pareja, las escuelas públicas entregarían una formación y capacitación de calidad similar 
que sus contrapartes. No encontramos, entonces, evidencia de que la gestión pública de las escuelas represente una desventaja en sí para los estudiantes. Esto no implica que la magra situación de la educación pública en Chile no sea insatisfactoria, respecto de lo que existe abundante evidencia comparativa, sino por el contrario implica que la educación particular subvencionada no está obteniendo un desempeño superior.

En segundo lugar, la modalidad de la escuela donde se estudia depende evidentemente de la posición social de origen de los individuos, y posee importantes efectos finales en el logro de las personas. Esto significa que la diferenciación de la educación, y en particular la diferenciación de la educación financiada públicamente, aporta importante rigidez al proceso de estratificación de la sociedad chilena y contribuye inapelablemente a la transmisión de las desigualdades que caracterizan al país. Estos efectos existen de modo adicional al ya muy fuerte impacto en las perspectivas de logro individual que posee la posición social de origen en Chile. Es decir, el modelo educativo chileno no sólo fracasa en reducir adecuadamente las brechas de oportunidades generadas en el ámbito de los hogares, sino que incrementa estos efectos a través de una oferta educacional socialmente segregada.

En tercer lugar, el proceso de municipalización de las escuelas públicas ha significado un desmedro de las oportunidades relativas de quienes acceden a éstas. Controlado el efecto en las posiciones ocupacionales que implica la transformación productiva del país y el desarrollo técnico en general, las personas que estudiaron en escuelas estatales obtienen sistemáticamente mejores resultados relativos que las personas que estudiaron en escuelas municipalizadas.

En cuarto lugar, el impacto de la modalidad escolar sobre el logro ocupacional se produce principalmente a través de la escolaridad, y secundariamente a través de la posición ocupacional inicial de los individuos. Esto implica que las personas que asisten a la educación municipalizada acumulan una desventaja durante el proceso educativo que no se asocia, directamente, al desempeño laboral. Si bien es posible que la menor escolaridad se asocie a déficits pedagógicos que dificulten el acceso a la educación superior, resulta llamativo que este tipo de déficits no se manifiesten también como efectos directos. Si sumamos a este indicio el hecho de que las escuelas particulares subvencionadas cuentan con ventajas para su desempeño como la posibilidad (fáctica) de seleccionar estudiantes, así como de agregar insumos a través de financiamientos complementarios, cabe hipotetizar que las desventajas acumuladas por los estudiantes de la educación pública se explican por otro tipo de mecanismos.

Estos mecanismos, posiblemente, se originen en la misma existencia de la segregación social de la educación: reducción de expectativas, educación funcionalmente segmentada, estratificación del acceso a redes y capital social, etc. Estos mecanismos quedan aún por ser investigados.

Cabe recordar que la diferenciación generada por las reformas educacionales al interior de la educación financiada públicamente no se restringe a las distinciones entre distintas modalidades educativas. El proceso de municipalización, por una parte, implica el acceso a escuelas "públicas" en condiciones de financiamiento completamente diferentes de acuerdo a la comuna de residencia de las personas, lo que genera toda una gama de dispositivos de transmisión de la desigualdad al interior de las escuelas municipalizadas. Por otra parte, diversos estudios demuestran que la educación particular subvencionada posee una oferta educativa extremadamente diferenciada según la posición social de los padres de sus 
estudiantes. Estas diferenciaciones no se reducen únicamente a la disposición de recursos adicionales, sino a la orientación escolar hacia objetivos socialmente segmentados: entre quienes reciben excelencia pedagógica y quienes reciben disciplinamiento (Castillo et al. 2011). Estas dos fuentes de mayor segregación social de las oportunidades a partir de la reforma educativa se asocian estrechamente con los importantes fenómenos de segregación espacial que caracterizan a Santiago de Chile. Evaluar estos efectos combinados es una deuda importante en esta línea de investigación que requerirá de información retrospectiva sobre los lugares de residencia de los individuos, que lamentablemente no estaba disponible en este caso.

En este sentido, los resultados no pueden más que mostrar una fracción de los efectos de la progresiva diferenciación interna de la educación pública sobre la desigualdad y su transmisión en Chile. En circunstancias de que este modelo educativo entra en cuestión, se requiere urgentemente de información retrospectiva y detallada que permita, por una parte, analizar estos fenómenos a la luz de las diferenciaciones internas a cada modalidad que se producen en el contexto de la segregación espacial de las ciudades, y por otra conocer los efectos de la carrera escolar en el tiempo a través de información detallada sobre el curso de vida escolar de las personas, lo que aportaría a despejar las dudas restantes sobre los mecanismos por los cuales la desigualdad educativa se transforma en nuevas (repetidas) desigualdades de clase.

Finalmente, queda comentar el hecho de que los resultados demuestran el efecto pernicioso en términos de desigualdad social de la educación particular subvencionada, al tiempo que reafirma el hallazgo cada vez más aceptado de que la competencia que caracterizaría supuestamente este último tipo de arreglo institucional no aporta, efectivamente, mejores resultados educativos a sus estudiantes (véase Elacqua, 2004; García-Huidobro y Bellei 2006; Hsieh y Urquiola 2003; Tokman 2002). En este sentido, desde una perspectiva de política pública, la persistencia de este modelo educativo sólo parece sostenible en pos de los intereses de los sostenedores particulares. En términos de valor social real, resulta completamente injustificable.

\section{REFERENCIAS BIBLIOGRÁFICAS}

Castillo, P., González, A. y Puga, I. (2011, en publicación). Gestión y efectividad en educación: Evidencias comparativas entre establecimientos municipales y particulares subvencionados. Estudios Pedagógicos.

Contreras, D., Cooper, R., Hermann, J. y Neilson, C. (2007). Movilidad y vulnerabilidad en Chile (Serie En Foco 56), Santiago de Chile: Expansiva.

Erikson, R. y Goldthorpe, J. (1992). The Constant Flux: a study of class mobility in industrial societies, Oxford: Clarendon Press.

Elacqua, G. (2004). El consumidor de la educación: El actor olvidado de la libre elección de colegios en Chile, Santiago de Chile: Universidad Adolfo Ibáñez.

García-Huidobro, J.E. y Bellei, C. (2006). ¿Remedio para la inequidad? La subvención escolar preferencial. Revista Mensaje, Marzo-Abril.

Ganzeboom, H. y Treiman, J. (1996). International Comparable Measures of Occupational Status for the 1988 ISCO. Social Science Research, vol. 25, 201-239.

Hsieh, C. y Urquiola, M. (2003). When Schools Compete, How do they Compete? An Assessment of Chile's Nationwide School Voucher Program. NBER Working Paper Nr. 10008. 
Larráñaga, O. (2004). Competencia y participación privada: la experiencia chilena en educación. Estudios Públicos, vol. 96, 107-144.

Núñez, J. y Risco, C. (2005). Movilidad intergeneracional del ingreso en Chile (Serie En Foco 58), Santiago de Chile: Expansiva.

Puga, I. y Solís, P. (2010). Estratificación y transmisión de la desigualdad en Chile y México. Un estudio empírico en perspectiva comparada. En: E. Cárdenas y F. Torche (Eds.): La movilidad social en México, México: CEEY.

Rodríguez, A. y Winchester, L. (2001). Santiago de Chile: Metropolización, globalización, desigualdad. EURE (Santiago), vol. 27, n. 80, 121-139.

Sabatini, F. y Wormald, G. (2004). Santiago de Chile bajo la Nueva Economía, 1980-2000: Crecimiento, Modernización, y Oportunidades de Integración Social. Santiago de Chile: Pontificia Universidad Católica de Chile.

Shavit, Y. y Muller, W. (1998). From School to Work. A Comparative Study of Educational Qualifications and Occupational Destinations, Oxford: Clarendon Press.

Torche, F. (2005a). Privatization Reform and Inequality of Educational Opportunity: The Case of Chile. Sociology of Education, vol. 78, n. 4, 316-343.

Torche, F. (2005b). Unequal but Fluid: Social Mobility in Chile in Comparative Perspective. American Sociological Review, vol. 70, n. 3, 422-450.

Tokman, A. (2002). Is private education better? Evidence from Chile. Banco Central de Chile, Working Papers n. 147. 